Journal of Mathematics and Statistics 3 (4): 220-221, 2007

ISSN 1549-3644

(C) 2007 Science Publications

\title{
On Characteristic Functions of First Order Theta Function
}

\author{
İsmet Yildiz
}

University of Bahcesehir Vocational School, Beşiktaş-İstanbul, Turkey

\begin{abstract}
In this study, a relation on the coefficients periods of first order theta function according to the period pair using the theta characteristic values is established.
\end{abstract}

Key words: First-theta function, characteristic, period pair

\section{INTRODUCTION}

Definition1: For $u \in C, \operatorname{Im} \tau \succ 0$ and characteristic $\left[\begin{array}{c}\varepsilon \\ \varepsilon^{\prime}\end{array}\right]$, the function defined as

$\theta\left[\begin{array}{c}\varepsilon \\ \varepsilon^{\prime}\end{array}\right](u, \tau)=\sum_{n=-\infty}^{\infty} \exp \left\{\left(n+\frac{\varepsilon}{2}\right)^{2} \pi i \tau+2 \pi i\left(n+\frac{\varepsilon}{2}\right)\left(u+\frac{\varepsilon^{\prime}}{2}\right\}\right.$

is called the first order theta function ${ }^{[1]}$.

Definition 2: A half-period is half of a period (in particular a complex vector), written

$\left(\begin{array}{l}\mu \\ \mu^{\prime}\end{array}\right)=\frac{1}{2}\left\{\begin{array}{l}\mu \\ \mu^{\prime}\end{array}\right\}=\frac{\mu^{\prime}}{2}+\frac{\mu \tau}{2}$

A reduced half-period is half of a period in which $\mu$ and $\mu^{\prime}$ where $\mu$ and $\mu^{\prime}$ are integers .

In the present paper, whenever the integers $\mu$ and $\mu^{\prime}$ will be as $\mu=1$ and $\mu^{\prime}=1$, unless otherwise stated $^{[2]}$.

In this study,

$\left[\begin{array}{l}\varepsilon \\ \mathcal{E}^{\prime}\end{array}\right] \equiv\left[\begin{array}{l}1 \\ 1\end{array}\right],\left[\begin{array}{l}1 \\ 0\end{array}\right],\left[\begin{array}{l}0 \\ 1\end{array}\right],\left[\begin{array}{l}0 \\ 0\end{array}\right](\bmod 2)$

values of characteristic are $\left[\begin{array}{c}\varepsilon \\ \varepsilon^{\prime}\end{array}\right]$ used. When the periodicity of the function $\theta_{\theta}\left[\begin{array}{l}\varepsilon \\ \varepsilon^{\prime}\end{array}\right](u, \tau)$ for $(1, \tau)$ period pair is examined

$\theta\left[\begin{array}{l}\varepsilon \\ \varepsilon^{\prime}\end{array}\right](u, \tau)=\sum_{n=-\infty}^{\infty} \exp \left\{\left(n+\frac{\varepsilon}{2}\right)^{2} \pi i \tau+2 \pi i\left(n+\frac{\varepsilon}{2}\right)\left(u+1+\frac{\varepsilon^{\prime}}{2}\right)\right\}$

$=\sum_{n=-\infty}^{\infty} \exp \left\{\left(n+\frac{\varepsilon}{2}\right)^{2} \pi i \tau+2 \pi i\left(n+\frac{\varepsilon}{2}\right)\left(u+\frac{\varepsilon^{\prime}}{2}\right)+n 2 \pi i+\pi i \varepsilon\right\}$

$=(-1)^{\tau} \theta\left[\begin{array}{l}\varepsilon \\ \varepsilon^{\prime}\end{array}\right](u, \tau)=\mu_{1} \theta\left[\begin{array}{l}\varepsilon \\ \varepsilon^{\prime}\end{array}\right](u, \tau) \quad$, for $\mu_{1}=(-1)^{\tau}$ $\theta\left[\begin{array}{l}\varepsilon \\ \varepsilon^{\prime}\end{array}\right](u+\tau, \tau)=\sum_{n=-\infty}^{\infty} \exp \left\{\left(n+\frac{\varepsilon}{2}\right)^{2} \pi i \tau+2 \pi i\left(n+\frac{\varepsilon}{2}\right)\left(u+\tau+\frac{\varepsilon^{\prime}}{2}\right)\right\}$

$=\sum_{n=-\infty}^{\infty} \exp \left\{\left(n+\frac{\varepsilon}{2}\right)^{2} \pi i \tau+2 \pi i\left(n+\frac{\varepsilon}{2}\right)\left(u+\frac{\varepsilon^{\prime}}{2}\right)+n 2 \pi i \tau+\pi i \tau \varepsilon\right\}$

$=(-1)^{\tau} \exp \{-\pi i \tau-2 \pi i u\} . \theta\left[\begin{array}{l}\varepsilon \\ \varepsilon^{\prime}\end{array}\right](u, \tau)$

If we choose $\mu_{2}=(-1)^{\tau} \exp \{-\pi i \tau-2 \pi i u\}$ then we obtain the following equality

$\theta\left[\begin{array}{l}\varepsilon \\ \varepsilon^{\prime}\end{array}\right](u+\tau, \tau)=\mu_{2} \theta\left[\begin{array}{l}\varepsilon \\ \varepsilon^{\prime}\end{array}\right](u, \tau)$

Hence

$\theta\left[\begin{array}{l}\varepsilon \\ \varepsilon^{\prime}\end{array}\right](u+1+\tau, \tau)=\sum_{n=-\infty}^{\infty} \exp \left\{\left(n+\frac{\varepsilon}{2}\right)^{2} \pi i \tau+2 \pi i\left(n+\frac{\varepsilon}{2}\right)\left(u+1+\tau+\frac{\varepsilon^{\prime}}{2}\right)\right\}$

$=\sum_{n=-\infty}^{\infty} \exp \left\{\left(n+\frac{\varepsilon}{2}\right)^{2} \pi i \tau+2 \pi i\left(n+\frac{\varepsilon}{2}\right)\left(u+\frac{\varepsilon^{\prime}}{2}\right)+n 2 \pi i \tau+\pi i \tau \varepsilon\right\}$

$=(-1)^{\tau} \exp \left\{-\pi i \tau-2 \pi i u-\pi i \varepsilon^{\prime}\right\} . \theta\left[\begin{array}{l}\varepsilon \\ \varepsilon^{\prime}\end{array}\right](u, \tau)$

By using

$\mu_{3}=(-1)^{\tau} \exp \left\{-\pi i \tau-2 \pi i u-\pi i \varepsilon^{\prime}\right\}$

we obtain

$\theta\left[\begin{array}{l}\varepsilon \\ \varepsilon^{\prime}\end{array}\right](u+1+\tau, \tau)=\mu_{3} \theta\left[\begin{array}{l}\varepsilon \\ \varepsilon^{\prime}\end{array}\right](u, \tau)$

As it is seen here, for $\mu_{3}=1$, because $\theta\left[\begin{array}{l}\varepsilon \\ \varepsilon^{\prime}\end{array}\right](u, \tau)$ is

doubly periodic, it would be an elliptic function.

Theorem: For $r \in \mathrm{N}^{+}$

$\theta\left[\begin{array}{c}\varepsilon \\ \varepsilon^{\prime}\end{array}\right]\left(u+\frac{1}{2^{r}}+\frac{\tau}{2^{r}}, \tau\right)=\exp \left\{-\frac{1}{4^{r}}(\tau+2) \pi i-\frac{1}{2^{r}}\left(2 u+\varepsilon^{\prime}\right) \pi i\right\} \theta\left[\begin{array}{c}\varepsilon+\frac{1}{2^{r-1}} \\ \varepsilon^{\prime}+\frac{1}{2^{r-1}}\end{array}\right](u, \tau)$

Proof

$\theta\left[\begin{array}{c}\varepsilon \\ \varepsilon^{\prime}\end{array}\right]\left(u+\frac{1}{2^{r}}+\frac{\tau}{2^{r}}, \tau\right)=\sum_{n=-\infty}^{\infty} \exp \left\{\left(n+\frac{\varepsilon}{2}\right)^{2} \pi i \tau+2 \pi i\left(n+\frac{\varepsilon}{2}\right)\left(u+\frac{1}{2^{r}}+\frac{\tau}{2^{r}}+\frac{\varepsilon^{\prime}}{2}\right)\right\}$

$=\sum_{n=-\infty}^{\infty} \exp \left\{\left(n+\frac{\varepsilon}{2}\right)^{2} \pi i \tau+2 \pi i\left(n+\frac{\varepsilon}{2}\right)\left(u+\frac{\varepsilon^{\prime}}{2}\right)+\left(\frac{n \pi i \tau}{2^{r-1}}+\frac{n \pi i}{2^{r-1}}+\frac{\pi i \tau \varepsilon}{2^{r}}+\frac{\pi i \varepsilon}{2^{r}}\right)\right\} \quad$ (2)

Also

Corresponding Author: $\quad$ İsmet Yıldız, University of Bahcesehir Vocational School, Beşiktaş-İstanbul, Turkey 
On the other hand

$$
\begin{aligned}
& \theta\left[\begin{array}{c}
\varepsilon+\frac{1}{2^{r-1}} \\
\varepsilon^{\prime}+\frac{1}{2^{r-1}}
\end{array}\right](u, \tau) \\
& =\sum_{n=-\infty}^{\infty} \exp \left\{\begin{array}{c}
\left(n+\frac{\varepsilon}{2}\right)^{2} \pi i \tau+2 \pi i\left(n+\frac{\varepsilon}{2}\right)\left(u+\frac{\varepsilon^{\prime}}{2}\right)+\frac{2 \pi i \tau}{2^{r-1}}+\frac{2 \pi i \varepsilon^{\prime}}{2^{r-1}}+\frac{n \pi i \tau}{2^{r-1}}+\frac{n \pi i \tau}{2^{r-1}} \\
+\frac{\pi i \tau \varepsilon}{2^{r}}+\frac{n \pi i}{2^{r-1}}+\frac{\pi i \tau}{4^{r}}+\frac{2 \pi i}{4^{r}}+\frac{\pi i \varepsilon}{2^{r}}
\end{array}\right\}
\end{aligned}
$$

and

$$
\begin{aligned}
& \exp \left\{-\frac{1}{4^{r}}(\tau+2) \pi \dot{\pi}-\frac{1}{2^{r}}\left(2 u+\varepsilon^{\prime}\right) \pi \dot{i}\right\} \theta\left[\begin{array}{c}
\varepsilon+\frac{1}{2^{r-1}} \\
\varepsilon^{\prime}+\frac{1}{2^{r-1}}
\end{array}\right](u, \tau) \\
& \left.=\sum_{n=-\infty}^{\infty} \exp \left\{\left(n+\frac{\varepsilon}{2}\right)^{2} \pi i \tau+2 \pi i\left(n+\frac{\varepsilon}{2}\right)\left(u+\frac{\varepsilon^{\prime}}{2}\right)+\frac{n \pi i \tau}{2^{r-1}}+\frac{n \pi i}{2^{r-1}}+\frac{\pi i \tau \varepsilon}{2^{r}}+\frac{\pi i \varepsilon}{2^{r}}\right)\right\}
\end{aligned}
$$

Using the equalities of (2) and (3) for

$$
\mu_{4}=\exp \left\{-\frac{1}{4^{r}}(\tau+2) \pi i-\frac{1}{2^{r}}\left(2 u+\varepsilon^{\prime}\right) \pi i\right\}
$$

we obtain the following equality

$$
\theta\left[\begin{array}{c}
\varepsilon \\
\varepsilon^{\prime}
\end{array}\right]\left(u+\frac{1}{2^{r}}+\frac{\tau}{2^{r}}, \tau\right)=\mu_{4} \theta\left[\begin{array}{c}
\varepsilon+\frac{1}{2^{r-1}} \\
\varepsilon^{\prime}+\frac{1}{2^{r-1}}
\end{array}\right](u, \tau)
$$

By the theorem given above we can obtain the following characteristic equalities for $\mathrm{u}=0$ value of the complex variable

$$
\begin{aligned}
& \text { (a) } \left.\begin{array}{l}
1 \\
1
\end{array}\right]\left(0+\frac{1}{2^{r}}+\frac{\tau}{2^{r}}, \tau\right)=\exp \left\{-\frac{1}{4^{r}}(\tau+2) \pi i-\frac{1}{2^{r}} \pi i\right\} \theta\left[\begin{array}{c}
1+\frac{1}{2^{r-1}} \\
1+\frac{1}{2^{r-1}}
\end{array}\right](0, \tau) \\
& =\sum_{n=-\infty}^{\infty} \exp \left\{\left(n+\frac{1}{2}+\frac{1}{2^{r}}\right)^{2} \pi i \tau+2 \pi i\left(n+\frac{1}{2}+\frac{1}{2^{r}}\right)\left(0+\frac{1}{2}+\frac{1}{2^{r}}\right)-\frac{\pi i \tau}{4^{r}}-\frac{\pi i}{2^{2 r-1}}-\frac{\pi i}{2^{r}}\right\} \\
& =\sum_{n=-\infty}^{\infty} \exp \left\{\left(n+\frac{1}{2}\right)^{2} \pi i \tau+\frac{n \pi i \tau}{2^{r-1}}+\frac{\pi i \tau}{2^{r}}+\frac{n \pi i}{2^{r-1}}+\frac{\pi i}{2^{r}}+\frac{\pi i}{2}+n \pi i\right\}(4) \\
& \theta\left[\begin{array}{l}
1 \\
1
\end{array}\right]\left(0+\frac{1}{2^{r}}+\frac{\tau}{2^{r}}, \tau\right)=\exp \left\{-\frac{1}{4^{r}}(\tau+2) \pi i-\frac{1}{2^{r}} \pi i\right\} \theta\left[\begin{array}{l}
1+\frac{1}{2^{r-1}} \\
0+\frac{1}{2^{r-1}}
\end{array}\right](0, \tau) \\
& =\sum_{n=-\infty}^{\infty} \exp \left\{\left(n+\frac{1}{2}+\frac{1}{2^{r}}\right)^{2} \pi i \tau+2 \pi i\left(n+\frac{1}{2}+\frac{1}{2^{r}}\right)\left(0+\frac{1}{2}+\frac{1}{2^{r}}\right)-\frac{\pi i \tau}{4^{r}}-\frac{\pi i}{2^{2 r-1}}\right\} \\
& =\sum_{n=-\infty}^{\infty} \exp \left\{\left(n+\frac{1}{2}\right)^{2} \pi i \tau+\frac{n \pi i \tau}{2^{r-1}}+\frac{\pi i \tau}{2^{r}}+\frac{n \pi i}{2^{r-1}}+\frac{\pi i}{2^{r}}+\frac{\pi i}{2}+n \pi i\right\}(5)
\end{aligned}
$$

From the equation (4) and (5), we can get the following equality

$$
\begin{aligned}
& \exp \left\{-\frac{1}{4^{r}}(\tau+2) \pi i-\frac{1}{2^{r}} \pi i\right\} \theta\left[\begin{array}{c}
1+\frac{1}{2^{r-1}} \\
1+\frac{1}{2^{r-1}}
\end{array}\right](0, \tau)=\exp \left\{-\frac{1}{4^{r}}(\tau+2) \pi i\right\} \theta\left[\begin{array}{c}
1+\frac{1}{2^{r-1}} \\
0+\frac{1}{2^{r-1}}
\end{array}\right](0, \tau) \\
& \text { (b) } \theta_{0}\left[\begin{array}{l}
0 \\
0
\end{array}\right]\left(0+\frac{1}{2^{r}}+\frac{\tau}{2^{r}}, \tau\right)=\exp \left\{-\frac{1}{4^{r}}(\tau+2) \pi i\right\} \theta\left[\begin{array}{c}
0+\frac{1}{2^{r-1}} \\
0+\frac{1}{2^{r-1}}
\end{array}\right](0, \tau)
\end{aligned}
$$

$$
\begin{aligned}
& =\sum_{n=-\infty}^{\infty} \exp \left\{\left(n+\frac{1}{2^{r}}\right)^{2} \pi i \tau+2 \pi i\left(n+\frac{1}{2^{r}}\right)\left(0+\frac{1}{2^{r}}\right)-\frac{\pi i \tau}{4^{r}}-\frac{\pi i}{2^{2 r-1}}\right\} \\
& =\sum_{n=-\infty}^{\infty} \exp \left\{n^{2} \pi i \tau+\frac{n \pi i \tau}{2^{r-1}}+\frac{n \pi i}{2^{r-1}}\right\} \\
& \theta\left[\begin{array}{l}
0 \\
1
\end{array}\right]\left(0+\frac{1}{2^{r}}+\frac{\tau}{2^{r}}, \tau\right)=\exp \left\{-\frac{1}{4^{r}}(\tau+2) \pi i-\frac{\pi i}{2^{r}}\right\} \theta\left[\begin{array}{c}
0+\frac{1}{2^{r-1}} \\
1+\frac{1}{2^{r-1}}
\end{array}\right](0, \tau) \\
& =\sum_{n=-\infty}^{\infty} \exp \left\{\left(n+\frac{1}{2^{r}}\right)^{2} \pi i \tau+2 \pi i\left(n+\frac{1}{2^{r}}\right)\left(0+\frac{1}{2}+\frac{1}{2^{r}}\right)-\frac{\pi i \tau}{4^{r}}-\frac{\pi i}{2^{2 r-1}}-\frac{\pi i}{2^{r}}\right\} \\
& =\sum_{n=-\infty}^{\infty} \exp \left\{n^{2} \pi i \tau+\frac{n \pi i \tau}{2^{r-1}}+n \pi i+\frac{n \pi i}{2^{r-1}}\right\}
\end{aligned}
$$

If $n=2 k \in \mathrm{N}^{+}$, then form the equalities (6) and (7) the following is obtained

$$
\exp \left\{-\frac{1}{4^{r}}(\tau+2) \pi i-\frac{\pi i}{2^{r}}\right\} \theta\left[\begin{array}{c}
0+\frac{1}{2^{r-1}} \\
1+\frac{1}{2^{r-1}}
\end{array}\right](0, \tau)=\exp \left\{-\frac{1}{4^{r}}(\tau+2) \pi i\right\} \theta\left[\begin{array}{c}
0+\frac{1}{2^{r-1}} \\
0+\frac{1}{2^{r-1}}
\end{array}\right](0, \tau)
$$

\section{CONCLUSION}

With the help of this theorem proved above, transformations among theta functions can be found for characteristic values $\left[\begin{array}{l}\varepsilon \\ \varepsilon^{\prime}\end{array}\right]$ according to all multiples $\frac{1}{2^{r}}$ of the periods.

For example; if $r=4$, then it follows that

$\theta\left[\begin{array}{c}\varepsilon \\ \varepsilon^{\prime}\end{array}\right]\left(u+\frac{1}{16}+\frac{\tau}{16}, \tau\right)=\exp \left\{-\frac{1}{256}(\tau+2) \pi i-\frac{1}{16}\left(2 u+\varepsilon^{\prime}\right) \pi i\right\} \theta\left[\begin{array}{c}\varepsilon+\frac{1}{8} \\ \varepsilon^{\prime}+\frac{1}{8}\end{array}\right](u, \tau)$

The subject that should be discussed here is; characteristic values $\left[\begin{array}{l}\varepsilon \\ \varepsilon^{\prime}\end{array}\right] \equiv\left[\begin{array}{l}1 \\ 1\end{array}\right],\left[\begin{array}{l}1 \\ 0\end{array}\right],\left[\begin{array}{l}0 \\ 1\end{array}\right],\left[\begin{array}{l}0 \\ 0\end{array}\right](\bmod 2)$ of first order theta function can be expressed as characteristic values $\theta\left[\begin{array}{l}\varepsilon+\frac{1}{2^{r-1}} \\ \varepsilon^{\prime}+\frac{1}{2^{r-1}}\end{array}\right]$

\section{REFERENCES}

1. Harry, E.R., 1973. Elliptic functions, Theta Functions and Riemann Surfaces. The Williams and Wilkins Company Baltimore, Maryland, pp: 75-79.

2. Yildiz, İ., 2004. On extension of the modular transformations over the modular group by reflection. Appl. Math. Comput., 153: 111-116.

3. Yild1z, İ., 2005. On the elliptic function arising from the theta functions and dedekind function. J. Math. \& Stat., 1: $153-159$. 